\title{
Associations of genetic variations in EYA4, GRHL2 and DFNA5 with noise-induced hearing loss in Chinese population: a case- control study
}

Xuhui Zhang ${ }^{1+}$, Yi Liu ${ }^{2 \dagger}$, Lei Zhang ${ }^{1}$, Zhangping Yang ${ }^{1}$, Luoxian Yang ${ }^{1}$, Xuchu Wang ${ }^{1}$, CaiXia Jiang ${ }^{1}$, Qiang Wang ${ }^{1}$, Yuyong Xia', Yanjuan Chen ${ }^{3}$, Ou Wu${ }^{1}$ and Yimin Zhu ${ }^{2^{*}}$

\begin{abstract}
Background: Both environmental and genetic factors are attributable to the incidence of noise-induced hearing loss $(\mathrm{NIHL})$. The purpose of this study was to examine the associations between genetic variations in the EYA4, GRHL2 and DFNA5 genes and the risk to noise-induced hearing loss (NIHL) in a Chinese population.

Methods: A case-control study was conducted with $476 \mathrm{NIHL}$ workers and 475 normal hearing workers matched with gender, years of noise exposure, and intensity of noise exposure. Twelve tag single-nucleotide polymorphisms (SNP) in the EYA4, GRHL2 and DFNA5 genes were genotyped using nanofluidic dynamic arrays on the Fluidigm platform. Multiple logistic regression was used to analyze the associations of genetic variations with NIHL adjusted by age, smoking/drinking status, and cumulative noise exposure and their interactions with noise exposure.

Results: The SNPs of rs3777781and rs212769 in the EYA4 gene were significantly associated with NIHL risk. In rs3777781, comparing with the subjects carrying with $\Pi$ types, the carriers with AT and AA genotypes had the decreased risk of $\mathrm{NIHL}(\mathrm{OR}=0.721,95 \% \mathrm{Cl}=0.522$ - 0.996). In rs212769, the $\mathrm{AG}$ and AA carriers had increased $\mathrm{NIHL}$ risk (OR $=1.430,95 \% \mathrm{Cl}=1.014$ - 2.016) compared with the subjects with $\mathrm{GG}$ genotype. Rs666026 in the associated GRHL2 gene and rs2521758 in the DFNA5 gene were marginally t associated with NIHL $(P=0.065$ and 0.052, respectively). Rs2521758 and rs212769 had significantly interacted with noise exposure $(P<0.05)$.

Conclusions: Genetic variations in the EYA4, GRHL2 and DFNA5 genes and their interactions with occupational noise exposure may play an important role in the incidence of $\mathrm{NIHL}$.
\end{abstract}

Keywords: Noise exposure, Genetic susceptibility, EYA4, GRHL2, DFNA5, Single-nucleotide polymorphism/SNP

\section{Introduction}

Noise is the most widespread environmental pollution that exposed in the occupational and living environment. Regular noise exposure leads to noise-induced hearing loss (NIHL), which is the most common occupational disease [1]. NIHL is a complex disease, resulted from the interaction of environmental and genetic risk factors [2]. Besides noise exposure, smoking, organic solvent exposure, higher blood pressure and cholesterol also increased the risk of NIHL [3]. However, the previous findings indicated

\footnotetext{
*Correspondence: zhuym@zju.edu.cn

${ }^{+}$Equal contributors

${ }^{2}$ Department of Epidemiology and Biostatistics, Zhejiang University School of Public Health, 388 Yu-Hang-Tang Road, Hangzhou 310058, Zhejiang, P.R.

China

Full list of author information is available at the end of the article
}

that the subjects had different degrees of NIHL risk even if they were exposed under similar noise environment. These findings implicated that the genetic susceptibility and its interaction with environmental factors might play important role in the development of NIHL $[4,5]$.

Animal experiments have proved that genetics contribute to the incidence of NIHL [6,7]. In human, the previous genetic studies have demonstrated that variants in $\mathrm{CDH} 23$ [8, 9], hOGG1 [10], catalase [11], heat shock protein 70 [12], KCNE1 and KCNQ4 [13] associated with the NIHL risk. Recently, we found that the genetic variants in the PCDH15 gene were associated with NIHL risk and this variant also modified the biological effect induced by occupational-noise exposure [14]. However, 
the known genetic polymorphisms only explained a small part of the etiology in NIHL.

The EYA4 and GRHL2 genes are transcription factors, and associated with composition of Corti [15]. The EYA4 gene is encoded for the protein of Eyes absent homolog 4 (EYA4). This protein acts through its protein phosphatase activity. It also may be important in eye development, and for continued function of the mature organ of Corti. Previous studies indicated that mutations in the $E Y A$ gene were associated with postlingual, progressive, autosomal dominant hearing loss at the deafness [16]. EYA4 localizess to the autosomal dominant non-syndromic hearing loss (NSHL) locus DFNA10 on chromosome 6q23. Several mutations in the EYA4 had been found to be associated with progressive and hearing loss [17-19]. The Grainyhead like 2 (GRHL2) gene is located on chromosome 8q22.3 and it is also a transcription factor cellular promoter 2-like 3. It is highly expressed in epithelial cells lining the cochlear Duct. GRHL2 plays an important role in epithelial tissues as well as in epithelial cell maintenance [20]. Van Laer et al. [21] found the genetic variants in GRHL2 gene was associated with age-related hearing impairment. Rs611419 in GRHL2 was also reported to be related with the risk of NIHL in Chinese population [22].

DFNA5 was firstly discovered to be associated with autosomal dominant hearing loss. A mutation in the DFNA5 gene was found to associated with autosomal dominant hereditary hearing loss [23]. DFNA5 contains two domains separated by a hinge region. The first domain in DFNA5 induces apoptosis when transfected into cell lines while the second domain may shield the apoptotic-inducing sequences residing in the first domain. Therefore, it is hypothesized that the mutations in DFNA5 might function on both of sensorineural hearing loss and carcinogenesis through activating the apoptosis [24].

Given that the important roles of EYA4, GRHL2 and DFNA5 in auditory system, we assumed that the genetic variants in these three genes might associate with the risk of NIHL. To examine this hypothesis, we genotyped 12 tagSNPs in the EYA4, GRHL2 and DFNA5 genes in 476 NIHL workers and 475 normal hearing workers, and analysised the associations of these SNPs with NIHL. We also explored the interaction effects among these SNPs and noise exposure.

\section{Methods}

\section{Subjects}

The design of this study were previously described in detail [14]. Briefly, subjects in this study included 476 NIHL workers and 475 normal hearing workers. All the subjects were recruited from a cross-sectional survey of 4419 occupational noise- exposed workers conducted between March 1, 2011 and December 31, 2012. In this survey, the workers were employed in the noise- exposed factories of mechanical equipment and household appliance manufacturing, steel construction, and cigarette production/packaging in Hangzhou city, Zhejiang province, China. Intensity of noise in the workplace was determined by a noise statistical analyzer (AWA6218; Westernization Instrument Technology Co., Ltd., Beijing, China). Noise exposure was evaluated with equivalent continuous $\mathrm{dB}$ (A)- weighted sound pressure levels $\left(\mathrm{L}_{\mathrm{EX}, 8 \mathrm{~h}}\right)$ according to Occupational Health Standard of the People's Republic of China: Measurement of Noise in the Workplace (GBZ/T 189.8-2007) (China, 2007). All the subjects received annual health examinations, including routine physical examination, pure tone audiometry (PTA), epidemiological investigation, and whole-blood collection. The inclusion criteria of subjects in this cross-sectional survey were as follows: (1) Working at noised exposed workplace and $\mathrm{L}_{\mathrm{EX}, 8 \mathrm{~h}}$ was $>85 \mathrm{~dB}$ (A); (2) Cumulative time of noise exposure of $>1$ year. Cumulative time of noise exposure of each worker was recorded according to the files of occupational health surveillance and verified with epidemiological interview; (3) Han ethnicity. The subjects were excluded if they had a family history of hearing loss or histories of the diseases such as otitis, other otological diseases, head injury, exposure to explosives, or ototoxic drug administration. The study protocol was approved by the Research Ethics Committees of Hangzhou Center for Disease Prevention and Control, Zhejiang, China.

\section{PTA and NIHL assessment}

After participants stopped noise exposure for $>12 \mathrm{~h}$, audiometry was carried out for each subject using a Madsen Voyager 522 audiometer (Madsen, Taastrup, Denmark) in a soundproof room with a background noise level of $<25 \mathrm{~dB}$ (A). Hearing thresholds of both ears were determined with the ascending method in 5-dB steps at frequencies of 500, 1000, 2000, 3000, 4000, and $6000 \mathrm{~Hz}$. All the evaluations were performed by trained physicians using standard procedures. In order to exclude the confounding effects by age and gender, the audiometric raw data were calibrated for the effects of age and gender on the basis of the Diagnostic Criteria of Occupational NIHL (Chinese National Criteria GBZ49-2007). The hearing threshold at high frequency (HTHF) by PTA was defined as the average at 3000, 4000, and $6000 \mathrm{~Hz}$ for each ear, and the hearing threshold at speech frequency (HTSF) was defined as the average at 500, 1000, and $2000 \mathrm{~Hz}$ for each ear.

\section{Physical examination and epidemiological investigation}

A physical examination was performed for each subject. Parameters such as height, weight, systolic and diastolic blood pressure levels were measured by trained physicians following a standard protocol. Face-to-face interview was used to collect epidemiological data using a structured 
questionnaire administered by trained professional physicians. The information in the questionnaire included demographic characteristics; smoking/drinking status; history of medical conditions and drug use; history of exposure to noise, vibration, and toxic chemicals in the workplace; health habits; use of ear protection for noise.

\section{Definitions of NIHL and control subjects}

NIHL group included the workers with normal hearing before exposure, $>1$ year of occupational noise exposure, and an HTHF $>40 \mathrm{~dB}$ of hearing level (HL). In order to exclude hearing loss induced by non-noise exposure, the workers were excluded from the study if their differences of HTHF between left and right ears were great then $35 \mathrm{~dB}(\mathrm{HL})$. The normal group included the workers with $>1$ year of occupational noise exposure, and hearing thresholds $<25 \mathrm{~dB}(\mathrm{HL})$ at each frequency. In order to control the environmental confounders, control subjects were individually matched with NIHL subjects according to the variables of gender, intensity of noise and years of noise exposure. Because the majority of the subjects in the cross-sectional study were males (about $91.7 \%$ ), the subjects in this study were restricted on males.

\section{SNP selection and genotyping}

Tag SNPs in the EYA4、DFNA5、GRHL2 genes were selected as tSNPs on the basis of the HapMap database and previous findings from the literature. The criteria for searching for tSNPs were as follows: MAF (minor allele frequency) of $\mathrm{CHB}>0.10$, and a linkage disequilibrium value of $r^{2}>0.8$. Twelve candidate SNPs were selected using these criteria: rs 2521758 , rs 2521768 in the DFNA5 gene, rs3777781, rs212769, rs3777849, rs465147, rs3777860 in the EYA4 gene, rs666026, rs471757, rs10955255, rs682769 and rs1981361 in the GRHL2 gene.

Whole-blood and serum samples were collected from each subject after an overnight fast. Genomic DNA was extracted from peripheral blood using the Toyobo MagExtractor Genomic DNA Purification Kit (Toyobo, Osaka, Japan) following the manufacturer's protocol. All the subjects were genotyped using nanofluidic dynamic arrays on the Fluidigm platform (South San Francisco, CA, USA) in Bio-X Institutes (Shanghai, China) [25]. Repeated control samples were set in every genotyping plate, and the concordance was $>99 \%$.

\section{Statistics}

Cumulative noise exposure (CNE) was calculated as $\mathrm{CNE}=10 \times \log \left(10^{\mathrm{SPL}} \times\right.$ years of noise exposure $)$, where SPL means the sound pressure level $[\mathrm{dB}(\mathrm{A})]$ of noise exposure. Continuous variables for the normal distribution were expressed as mean \pm standard deviation (SD) and as median (P25, P75) for skewed distribution. Categorical variables were expressed as frequencies (\%). Student's $t$ test was used to examine the statistical significance for continuous variables, and the $\chi^{2}$ test was used for categorical variables. Hardy-Weinberg equilibrium were tested using Pearson's $\chi^{2}$ for each SNP among control subjects, and the SNPs with deviating from Hardy-Weinberg equilibrium were excluded from the analysis. Multiple logistic regression was used to calculate the OR and $95 \% \mathrm{CI}$, and gene-environmental interactions modified by confounders such as age, smoking/drinking status, and Cumulative noise exposure (CNE). All statistical analyses were performed using SPSS 19.0 for Windows (IBM Corporation, Armonk, NY, US).

\section{Results}

\section{Basic characteristics of the subjects}

The basic characteristics of the subjects have been described in detail in our previous study [14]. Briefly, the subjects included 476 NIHL subjects and 475 control subjects. All the subjects were males. The mean age was $36.6 \pm 8.5$ years in NIHL subjects, which was significant elder than that in control subjects $(32.8 \pm 8.0)(P<0.001)$. No significant differences were found between NIHL and normal hearing groups in the distributions of smoking and drinking status, years of noise exposure, median of noise intensities $(P>0.05)$. The median of cumulative noise exposure (CNE) in the NIHL group [95.5 (91.5, 100.5)] was a little, but not significantly higher than that in the normal hearing group $[94.3(91.0,97.8)](P>0.05)$.

\section{Associations of EYA4, GRHL2 and DFNA5variants with the risks of NIHL}

Basic information of SNPs in these three genes and the significance are shown in Table 1. Nine of studied SNPs in controls were in Hardy-Weinberg equilibrium distribution $(P>0.05)$, except for rs3777849, rs471757 and rs682769, which were excluded in the analysis.

Table 2 shows the odds ratios (ORs) of genotypes in the associated SNPs. The frequency of AT/AA in rs3777781 in NIHL group was $64.8 \%$, which was significantly lower than that in control group $(70.8 \%)(P<0.05)$. Comparing with the subjects with homozygotes of wild type (TT), the subjects carrying with variant A allele (AT and AA) decreased the risk of NIHL with the OR of $0.721(95 \%$ $\mathrm{CI}=0.522-0.996$ ). In rs212769, the percentage of the genotypes with AG and AA was $29.0 \%$ in the NIHL group and $24.8 \%$ in control group $(P<0.05)$. The subjects carrying with A allele (AG or AA) increased the risk of NIHL with an adjusted OR of $1.430(95 \% \mathrm{CI}=$ 1.014 - 2.016) comparing with the subjects with homozygous wildtype (GG). We also found that genotypes of rs666026 and rs2521758 had the marginal associations with NIHL ( $P=0.065$ and 0.052 , respectively). 
Table 1 Distributions of allele and genotype in the subjects of $\mathrm{NIHLand}$ normal hearing groups

\begin{tabular}{llllll}
\hline gene & SNP & A1/A2 & maf & case $^{a}$ & control $^{a}$ \\
\hline EYA4 & rs3777781 & A $T$ & 0.430 & $72 / 235 / 166$ & $98 / 236 / 137$ \\
& rs212769 & A/G & 0.141 & $8 / 129 / 336$ & $5 / 112 / 354$ \\
& rs3777849\# & A/G & 0.353 & $70 / 175 / 228$ & $85 / 182 / 204$ \\
& rs465147 & T/C & 0.010 & $0 / 7 / 465$ & $0 / 11 / 459$ \\
& rs3777860 & A/G & 0.355 & $62 / 217 / 194$ & $57 / 215 / 200$ \\
GRHL2 & rs666026 & G/T & 0.298 & $46 / 204 / 222$ & $40 / 186 / 244$ \\
& rs471757\# & T/C & 0.431 & $89 / 217 / 167$ & $105 / 208 / 158$ \\
& rs10955255 & G/A & 0.220 & $16 / 164 / 292$ & $23 / 173 / 275$ \\
& rs682769\# & A/G & 0.191 & $10 / 155 / 308$ & $11 / 163 / 297$ \\
& rs1981361 & A/G & 0.289 & $36 / 200 / 237$ & $34 / 205 / 232$ \\
DFNA5 & rs2521758 & G/T & 0.019 & $0 / 14 / 458$ & $1 / 20 / 450$ \\
& rs2521768 & C/T & 0.209 & $19 / 148 / 305$ & $27 / 155 / 289$ \\
\hline
\end{tabular}

apresented as the order of $A 1 A 1 / A 1 A 2 / A 2 A 2$. A1: the minor allele; $A 2$ : the major allele

${ }^{\#} P$ values $<0.05$ after Hardy-Weinberg equilibrium tests
Interaction and stratification analysis of EYA4, GRHL2 and DFNA5 by noise intensity and CNE

After adjusted by age, drinking, smoking status, significant multiplicative interactions for NIHL were found between rs2521758 and $\mathrm{CNE}\left(P=0.040, \mathrm{OR}_{\mathrm{int}}=0.794\right.$, $95 \% \mathrm{CI}=0.638-0.989)$, and between $\mathrm{rs} 212769$ and noise intensity $\left(P=0.041, \mathrm{OR}_{\mathrm{int}}=1.100,95 \% \mathrm{CI}=1.004-1.205\right)$ (Table 3). In the noise exposure with $\mathrm{CNE}<95$, the subjects with the genotypes of GT and GG in rs2521758 were found to have the decreased NIHL risk $(\mathrm{OR}=0.115,95 \%$ $\mathrm{CI}=0.014-0.921)$. However, no significant association of rs2521758 was found in the noise exposure with CNE >95 $(P>0.05)$. The subjects carrying A allele (AG or AA) in rs212769 had significantly increased the risk of NIHL in the noise intensity of $\geq 88 \mathrm{~dB}(\mathrm{~A})$ with the OR of 1.727 (95\% CI $=1.009-2.954)$, not significantly in the noise intensity of $<88 \mathrm{~dB}(\mathrm{~A})$.

\section{Discussion}

This study examined the associations of 12 SNPs in EYA4, GRHL2 and DFNA5 genes with the risk of NIHL. We found that the genetic variations of rs3777781,

Table 2 Associations of candidate SNPs with the risk of NIHL

\begin{tabular}{|c|c|c|c|c|c|c|}
\hline Gene & SNP & Genotype & Control n (\%) & Case n (\%) & P-value & OR $(95 \% \mathrm{Cl})^{a}$ \\
\hline \multirow[t]{10}{*}{ EYA4 } & rs3777781 & $\pi$ & $137(29.2)$ & $166(35.2)$ & & 1 \\
\hline & & AT & $236(50.3)$ & $235(49.8)$ & 0.116 & $0.759(0.539,1.070)$ \\
\hline & & $\mathrm{AA}$ & $96(20.5)$ & $71(15.0)$ & 0.015 & $0.570(0.363,0.895)$ \\
\hline & & $P_{\text {trend }}$ & & & 0.019 & \\
\hline & & AT/AA & $332(70.8)$ & $306(64.8)$ & 0.047 & $0.721(0.522,0.996)$ \\
\hline & rs212769 & GG & $354(75.2)$ & $336(71.0)$ & & 1 \\
\hline & & $A G$ & $112(23.8)$ & $129(27.3)$ & 0.062 & $1.395(0.984,1.979)$ \\
\hline & & AA & $5(1.1)$ & $8(1.7)$ & 0.214 & $2.369(0.608,9.234)$ \\
\hline & & $P_{\text {trend }}$ & & & 0.033 & \\
\hline & & $A G / A A$ & $117(24.8)$ & $137(29.0)$ & 0.041 & $1.430(1.014,2.016)$ \\
\hline \multirow[t]{5}{*}{ GRHL2 } & rs666026 & $\pi$ & $244(51.9)$ & $222(47.0)$ & & 1 \\
\hline & & GT & 186 (39.6) & $204(43.2)$ & 0.118 & $1.287(0.938,1.767)$ \\
\hline & & GG & $40(8.5)$ & $46(9.7)$ & 0.121 & $1.554(0.891,2.71)$ \\
\hline & & $P_{\text {trend }}$ & & & 0.051 & \\
\hline & & GT/GG & $226(48.1)$ & $250(53.0)$ & 0.065 & $1.329(0.983,1.798)$ \\
\hline \multirow[t]{9}{*}{ DFNA5 } & rs2521768 & $\pi$ & $289(61.4)$ & $305(64.6)$ & 0.158 & 1 \\
\hline & & CT & $155(32.9)$ & $148(31.4)$ & 0.249 & $0.825(0.595,1.144)$ \\
\hline & & CC & $27(5.7)$ & $19(4.0)$ & 0.089 & $0.524(0.249,1.104)$ \\
\hline & & $P_{\text {trend }}$ & & & 0.065 & \\
\hline & & $\mathrm{CT} / \mathrm{CC}$ & $182(38.6)$ & $167(35.4)$ & 0.124 & $0.782(0.571,1.070)$ \\
\hline & rs2521758 & $\pi$ & $450(95.5)$ & $458(97.0)$ & & 1 \\
\hline & & GT & $20(4.2)$ & $14(3.0)$ & 0.078 & $0.474(0.206,1.088)$ \\
\hline & & GG & $1(0.2)$ & $0(0.0)$ & 1.000 & \\
\hline & & GT/GG & $21(4.5)$ & $14(3.0)$ & 0.052 & $0.441(0.194,1.006)$ \\
\hline
\end{tabular}

${ }^{a}$ calculated with logistic regression adjusted by age, CNE, smoking, drinking 
Table 3 Stratified analysis of associated SNPs by CNE and intensity of noise exposure

\begin{tabular}{|c|c|c|c|c|c|c|c|}
\hline Exposure & SNP & Genotype & Control & Case & P1 & P2 & OR $(95 \% \mathrm{Cl})$ \\
\hline \multicolumn{8}{|l|}{ CNE } \\
\hline \multirow[t]{2}{*}{$<95$} & rs2521758 & $\pi$ & 244 & 209 & 0.040 & & 1 \\
\hline & & GT/GG & 12 & 3 & & $0.042^{\mathrm{a}}$ & $0.115(0.014,0.921)^{a}$ \\
\hline \multirow[t]{2}{*}{$\geq 95$} & & $\pi$ & 205 & 232 & & & 1 \\
\hline & & GT/GG & 9 & 10 & & $0.587^{\mathrm{a}}$ & $0.761(0.284,2.042)^{a}$ \\
\hline \multicolumn{8}{|c|}{ Intensity (dB) } \\
\hline \multirow[t]{2}{*}{$<88$} & rs212769 & GG & 211 & 181 & 0.041 & & 1 \\
\hline & & $\mathrm{AG} / \mathrm{AA}$ & 72 & 71 & & $0.503^{b}$ & $1.169(0.740,1.849)^{\mathrm{b}}$ \\
\hline \multirow[t]{2}{*}{$\geq 88$} & & GG & 143 & 155 & & & 1 \\
\hline & & $\mathrm{AG} / \mathrm{AA}$ & 45 & 66 & & $0.046^{\mathrm{b}}$ & $1.727(1.009,2.954)^{b}$ \\
\hline
\end{tabular}

P1: $P$ value of multiplicative interaction, which calculated by logistic model Multiplicative term; $P 2: P$ value of the association in each stratum

${ }^{a}$ calculated with logistic regression adjusted by age, smoking, drinking

${ }^{\mathrm{b}}$ calculated with logistic regression adjusted by age, smoking, drinking, years to noise exposure

rs212769, rs666026 and rs2521758 were associated with NIHL risk. Rs2521758 and rs212769 had interacted with noise exposure. These findings suggested that genetic polymorphisms in EYA4, GRHL2 and DFNA5 and their interactions with noise exposure might play important roles in NIHL incidence.

Konings et al. [26] had demonstrated that the SNPs in the EYA4 gene were associated with NIHL risk in Sweden and Poland populations. In this study, we investigated four SNPs in the EYA4 gene and replicated that rs3777781 and rs212769 were associated with NIHL risk in Chinese population. Furthermore, interaction effect was found between genotype of rs212769 and intensity of noise exposure. After stratification, significant association was only found in the intensity of noise $\geq 88 \mathrm{~dB}$ and non significance in the intensity of noise $<88 \mathrm{~dB}$. The combined effect of rs212769 and intensity of noise was 1.710 (1.115 - 2.623). To our knowledge, this is the first study to investigate the association of SNPs in EYA4 gene in Chinese population. In European, two intronic SNPs in the EYA4 gene was found to be significantly associated with age-related hearing impairment (ARHI). Rs212765 had reached the significance of 0.000565 with ARHI [21]. Although the biological mechanism is unclear, these findings suggested that genetic variation may be associated with the NIHL risk. EYA4 is a transcriptional activator and plays an important in eye development, and for continued function of the mature organ of Corti. Previous studies mainly focused on the mutations in EYA4 on hearing loss and had found that several causative mutations caused non-syndromic hearing loss [16-18, 20]. Together the evidences of present study with Kongs' study, it indicated that genetic variation in $E Y A$ gene might be a biomarker of genetic susceptibility for NIHL.

GRHL2 is known as a transcription factor cellular promoter 2-like 3 and highly expressed in epithelial cells lining the cochlear duct. It acts a critical role in embryonic development and maintains epithelial cells throughout life [20]. Animal study indicated GRHL2 involved in otic development and hearing. The mutation in this gene associated with hearing loss in humans. As a transcription activator, GRHL2 regulates apical junctional proteins and desmosomal cadherin expression. Peters et al. firstly reported that mutation in GRHL2 could induce DFNA28 [20]. In European, genetic variation of GRHL2 associated with ARHI. Rs10955255 had arrived the significance of $8.38 \times 10^{-5}$ with ARHI [21]. For genetic susceptibility of NIHL, rs1981361 in Sweden and rs682769 in Poland population were found to be associated with NIHL [11]. However, we didn't observe the associations of these SNPs in the present study. We found marginal significance of rs666026 $(P=0.051)$, which was no significance in Sweden and Poland populations . Li et al. also found the significant association of rs611419 in Chinese population and the AT/TT genotypes conferred decreased NIHL risk [22]. These results might reflect the race difference in genetic susceptibility.

DFNA5 was first discovered in an extended Dutch family with autosomal dominant nonsyndromic hereditary HL $[27,28]$. Four mutations in introns 7 or 8 of DFNA5 have been reported to cause HL [29]. These four mutations lead to skipping of exon 8 , resulting in a frameshift, and presumably causing premature truncation of the DFNA5 protein. It was suggested that DFNA5-associated HL represents a mechanism of gain-of-function. DFNA5 contains two domains separated by a hinge region. The first domain in DFNA5 induces apoptosis when transfected into cell lines while the second domain may shield the apoptotic- inducing sequences residing in the first domain. It is a well known that apoptosis contributes to several acquired forms of hearing impairment. Apoptosis is a key contributor to the development of presbycusis, age-related hearing loss and cancer [24]. Apoptosis contributes to the pathology of acquired hearing impairment and to genetic 
hearing impairment [30]. The mutation in DFNA5 leads to a type of hearing loss that closely resembles the frequently observed age related hearing impairment (ARHI). However, no significant associations of four coding SNPs in GRHL2 gene were found to be related with ARHI [31]. Rs2521772 associated with NIHL in Sweden population, however, no significance in Poland population. In this study, we found the genotypes of rs2521758 had significant association with NIHL although its MAF is only 0.019 . These results indicated that genetic variation in GRHL2 gene might associated with NIHL risk.

This study was carried out based on a cross-sectional study on occupational noise-exposed worker. We recruited NIHL cases and sex, intensity of noise and years of noise exposure matched healthy controls under similar environmental exposure. Therefore, there were high comparability between NIHL cases and controls. We have also excluded the subjects whose audio-impairment were caused by risk factors of non-occupational exposure. The difference between two groups reflected the potential effect of genetic susceptibility. However, there were some limitations in this study. Due to the majority of the noise-exposed workers were males, therefore, the subjects in this study were also restricted to males. Although the sample size was relatively large in the previous studies, however, due to the lower biological effects of an individual SNP, the power of statistics might not be enough. On the other hand, the significance could not arrived at the multiple correction. In order to decrease the false positive and negative errors, the results should be validated in other independent population with large sample size including females.

\section{Conclusion}

We found that SNPs of rs3777781, rs212769, rs666026 and rs2521758 were associated with NIHL risk. Interaction effects were found between rs2521758, rs212769 and noise exposure. These findings suggested that genetic variations in EYA4, GRHL2 and DFNA5 and their interactions with noise exposure may play important roles in NIHL incidence.

\section{Abbreviations}

SNP: Single-nucleotide polymorphism; Cl: Confidence interval; NIHL: Noiseinduced hearing loss; OR: Odds ratio; MAF: Minor allele frequency; CHB: Han Chinese individuals from Beijing; CNE: Cumulative noise exposure.

\section{Competing interests}

The authors declare that they have no competing interests.

\section{Authors' contributions}

$X Z$ and $Y Z$ conceived and designed the project. $Y Z, Y L$ drafted and refined the manuscript. $Y L$ performed laboratorial determination and statisitcs. $L Z$, $Y Z, Y L, X W, C J, Q W, O W, Y X$ and YC participated in the epidemiological investigation. All authors approved the final version of the paper for submission.

\section{Acknowledgements}

This study was supported by Science and Technology Project of Zhejiang Province (2015C33255), Hangzhou Medical \& Health Science and Technology Foundation (20110733Q19 and 20130633B38), the National Natural Science Foundation of China (81102200) and Zhejiang Provincial Program for the Cultivation of High-Level Innovative Health Talents.

\section{Author details}

${ }^{1}$ Hangzhou Center for Disease Control and Prevention, Hangzhou 310021, P.R. China. ${ }^{2}$ Department of Epidemiology and Biostatistics, Zhejiang University School of Public Health, 388 Yu-Hang-Tang Road, Hangzhou 310058, Zhejiang, P.R. China. ${ }^{3}$ Hangzhou Hospital for Prevention and Treatment of Occupational Diseases, Hangzhou 310014, Zhejiang, P.R. China.

Received: 14 July 2015 Accepted: 9 September 2015

Published online: 24 September 2015

\section{References}

1. Nelson DI, Nelson RY, Concha-Barrientos M, Fingerhut M. The global burden of occupational noise-induced hearing loss. Am J Ind Med. 2005;48(6):446-58.

2. Sliwinska-Kowalska M, Pawelczyk M. Contribution of genetic factors to noise-induced hearing loss: a human studies review. Mutat Res. 2013;752(1):61-5.

3. Rabinowitz PM, Galusha D, Slade MD, Dixon-Ernst C, O'Neill A, Fiellin M, et al. Organic solvent exposure and hearing loss in a cohort of aluminium workers. Occup Environ Med. 2008;65(4):230-5.

4. Konings A, Van Laer L, Van Camp G. Genetic studies on noise-induced hearing loss: a review. Ear Hear. 2009;30(2):151-9.

5. Henderson D, Subramaniam M, Boettcher FA. Individual Susceptibility to Noise-Induced Hearing-Loss - an Old Topic Revisited. Ear Hear. 1993;14(3):152-68

6. White CH, Ohmen JD, Sheth S, Zebboudj AF, McHugh RK, Hoffman LF, et al. Genome-wide screening for genetic loci associated with noise-induced hearing loss. Mamm Genome. 2009;20(4):207-13.

7. Davis RR, Newlander JK, Ling X, Cortopassi GA, Krieg EF, Erway LC. Genetic basis for susceptibility to noise-induced hearing loss in mice. Hear Res. 2001;155(1-2):82-90.

8. Sliwinska-Kowalska M, Noben-Trauth K, Pawelczyk M, Kowalski TJ. Single nucleotide polymorphisms in the Cadherin $23(\mathrm{CDH} 23)$ gene in Polish workers exposed to industrial noise. Am J Hum Biol. 2008;20(4):481-3.

9. Kowalski TJ, Pawelczyk M, Rajkowska E, Dudarewicz A, Sliwinska-Kowalska M. Genetic Variants of CDH23 Associated With Noise-Induced Hearing Loss. Otol Neurotol. 2014;35(2):358-65.

10. Shen HX, Cao JL, Hong ZQ, Liu K, Shi J, Ding L, et al. A Functional Ser326Cys Polymorphism in hOGG1 Is Associated with Noise-Induced Hearing Loss in a Chinese Population. Plos One. 2014;9(3):e89662.

11. Konings A, Van Laer L, Pawelczyk M, Carlsson PI, Bondeson ML, Rajkowska E, et al. Association between variations in CAT and noise-induced hearing loss in two independent noise-exposed populations. Hum Mol Genet. 2007;16(15):1872-83.

12. Konings A, Van Laer L, Michel S, Pawelczyk M, Carlsson PI, Bondeson ML, et al. Variations in HSP70 genes associated with noise-induced hearing loss in two independent populations. Eur J Hum Genet. 2009;17(3):329-35.

13. Pawelczyk M, Van Laer L, Fransen E, Rajkowska E, Konings A, Carlsson PI, et al. Analysis of Gene Polymorphisms Associated with K+ Ion Circulation in the Inner Ear of Patients Susceptible and Resistant to Noise-induced Hearing Loss. Ann Hum Genet. 2009;73:411-21.

14. Zhang XH, Liu Y, Zhang L, Yang ZP, Shao YX, Jiang CX, et al. Genetic variations in protocadherin 15 and their interactions with noise exposure associated with noise-induced hearing loss in Chinese population. Environ Res. 2014;135:247-52.

15. Lu JQ, Cheng XR, Li YQ, Zeng L, Zhao YM. Evaluation of individual susceptibility to noise-induced hearing loss in textile workers in China. Arch Environ Occup H. 2005;60(6):287-94.

16. Schonberger J, Wang L, Shin TJ, Kim SD, Depreux FFS, Zhu H, et al. Mutation in the transcriptional coactivator EYA4 causes dilated cardiomyopathy and sensorineural hearing loss. Nat Genet. 2005;37(4):418-22.

17. Wayne S, Robertson NG, DeClau F, Chen N, Verhoeven K, Prasad S, et al. Mutations in the transcriptional activator EYA4 cause late-onset deafness at the DFNA10 locus. Hum Mol Genet. 2001;10(3):195-200. 
18. Hildebrand MS, Coman D, Yang T, Gardner RJM, Rose E, Smith RJH, et al. A novel splice site mutation in EYA4 causes DFNA10 hearing loss. Am J Med Genet A. 2007;143A(14):1599-604

19. Hildebrand MS, Coman D, Yang T, Gardner RJ, Rose E, Smith RJ, et al. A novel splice site mutation in EYA4 causes DFNA10 hearing loss (vol 143, pg 1599, 2007). Am J Med Genet A. 2008;146A(8):1099.

20. Peters LM, Anderson DW, Griffith AJ, Grundfast KM, San Agustin TB, Madeo AC, et al. Mutation of a transcription factor, TFCP2L3, causes progressive autosomal dominant hearing loss, DFNA28. Hum Mol Genet. 2002;11(23):2877-85.

21. Van Laer L, Van Eyken E, Fransen E, Huyghe JR, Topsakal V, Hendrickx JJ, et al. The grainyhead like 2 gene (GRHL2), alias TFCP2L3, is associated with age-related hearing impairment. Hum Mol Genet. 2008;17(2):159-69.

22. Li X, Huo X, Liu K, Li X, Wang M, Chu H, et al. Association between genetic variations in GRHL2 and noise-induced hearing loss in Chinese high intensity noise exposed workers: a case-control analysis. Ind Health. 2013;51(6):612-21.

23. Nishio A, Noguchi Y, Sato T, Naruse TK, Kimura A, Takagi A, et al. A DFNA5 Mutation Identified in Japanese Families with Autosomal Dominant Hereditary Hearing Loss. Ann Hum Genet. 2014;78(2):83-91.

24. de Beeck KO, Van Camp G, Thys S, Cools N, Callebaut I, Vrijens K, et al. The DFNA5 gene, responsible for hearing loss and involved in cancer, encodes a novel apoptosis-inducing protein. Eur J Hum Genet. 2011;19(9):965-73.

25. Wang J, Lin M, Crenshaw A, Hutchinson A, Hicks B, Yeager M, et al. High-throughput single nucleotide polymorphism genotyping using nanofluidic Dynamic Arrays. BMC Genomics. 2009;10:561.

26. Konings A, Van Laer L, Wiktorek-Smagur A, Rajkowska E, Pawelczyk M, Carlsson PI, et al. Candidate gene association study for noise-induced hearing loss in two independent noise-exposed populations. Ann Hum Genet. 2009;73(2):215-24.

27. Van Laer L, Huizing EH, Verstreken M, van Zuijlen D, Wauters JG, Bossuyt PJ, et al. Nonsyndromic hearing impairment is associated with a mutation in DFNA5. Nat Genet. 1998;20(2):194-7.

28. Yu C, Meng X, Zhang S, Zhao G, Hu L, Kong X. A 3-nucleotide deletion in the polypyrimidine tract of intron 7 of the DFNA5 gene causes nonsyndromic hearing impairment in a Chinese family. Genomics. 2003;82(5):575-9.

29. Park HJ, Cho HJ, Baek Jl, Ben-Yosef T, Kwon TJ, Griffith AJ, et al. Evidence for a founder mutation causing DFNA5 hearing loss in East Asians. J Hum Genet. 2010;55(1):59-62.

30. de Beeck KO, Schacht J, Van Camp G. Apoptosis in acquired and genetic hearing impairment: the programmed death of the hair cell. Hear Res. 2011;281(1-2):18-27

31. Van Laer L, DeStefano AL, Myers RH, Flothmann K, Thys S, Fransen E, et al. Is DFNA5 a susceptibility gene for age-related hearing impairment? Eur J Hum Genet. 2002;10(12):883-6

\section{Submit your next manuscript to BioMed Central and take full advantage of:}

- Convenient online submission

- Thorough peer review

- No space constraints or color figure charges

- Immediate publication on acceptance

- Inclusion in PubMed, CAS, Scopus and Google Scholar

- Research which is freely available for redistribution

Submit your manuscript at www.biomedcentral.com/submit 